\title{
New Massive Gravity and $\mathrm{AdS}_{4}$ counterterms
}

\author{
Dileep P. Jatkar ${ }^{1 *}$ and Aninda Sinha ${ }^{2 \dagger}$ \\ ${ }^{1}$ Harish-Chandra Research Institute \\ Chhatnag Road, Jhusi, Allahabad, 211019, India \\ ${ }^{2}$ Centre for High Energy Physics, Indian Institute of Science, \\ C. V. Raman Avenue, Bangalore 560012, India
}

October 6, 2018

\begin{abstract}
We show that the recently proposed DBI extension of new massive gravity emerges naturally as a counterterm in $\mathrm{AdS}_{4}$. The resulting on-shell Euclidean action is independent of the cutoff at zero temperature. We also find that the same choice of counterterm gives the usual area law for the $\mathrm{AdS}_{4}$ Schwarzschild black hole entropy in a cut-off independent manner. The parameter values of the resulting counterterm action correspond to a $c=0$ theory in the context of $\mathrm{AdS}_{3} / \mathrm{CFT}_{2}$. We rewrite this theory in terms of the gauge field that is used to recast 3D gravity as a Chern-Simons theory.
\end{abstract}

*email: dileep@hri.res.in

$\dagger$ †email: asinha@cts.iisc.ernet.in 
New massive gravity(NMG) [1] is an interesting higher derivative theory of gravity in $2+1$ dimensions and has been the subject of much interest in recent times. The propagating degree of freedom in this theory is a massive spin-2 field. Interestingly, a different derivation based on the AdS/CFT correspondence was provided in [2]. Assuming that a dual conformal field theory exists, one can demand the existence of a c-theorem which posits the existence of a monotonic function in the space of couplings as the theory flows from the UV to the IR. It is the existence of a simple c-function that made this derivation possible in [2. By demanding the existence of a simple c-function [3], this theory can be extended to more than four derivative order [2, 4]. Of course one can ask if there are any interesting theories which are not truncated at a particular order, since any such truncation raises the obvious question as to what happens to higher order terms.

An interesting infinite order extension of gravity is Dirac-Born-Infeld (DBI) gravity. This theory agrees with NMG to quadratic orden when a weak field expansion is made. Quite remarkably, the cubic and quartic terms of this theory agree with the values obtained from the AdS/CFT calculation leading to a simple c-function [6, 7]. It was shown in [7, that the DBI-NMG theory also admits a simple c-function. Further properties of this theory have been investigated in [8].

Unfortunately, apart from the usual two derivative theory or $f(R)$ theories, it is known that there is a conflict between the unitarity of the bulk gravity theory and that of the boundary CFT [9, 10, 11]. It is quite possible that only the $c=0$ theories are to be taken seriously in this context. The relevant CFT may be a logarithmic CFT. There has been some evidence to this effect in the literature [9, 12, 13, 14. Although these higher derivative theories are interesting in their own right, a fundamental explanation for their origin is still lacking.

In this letter, we will make two interesting observations about NMG. Firstly, by examining counterterms in $\mathrm{AdS}_{4}$ with different boundary topologies and demanding that the total Euclidean action is independent of the cut-off, we will find that the boundary counterterm coincides with the DBI extension of NMG. The same counterterm leads to an area law for entropy for the Schwarzschild black hole in a cut-off independent manner. Further, it turns out that precisely for this choice of parameter values the DBI-NMG action in $2+1$ dimensions would correspond to a $c=0 \mathrm{CFT}$ in $1+1$ dimensions. Secondly, we will find a simple way to rewrite this DBI-NMG action in terms of the gauge field that is used to recast $2+1$ dimensional Einstein gravity into a Chern-Simons theory [15, 16].

It is well known that in order to make sense of the bulk action in the AdS/CFT correspondence, one needs to add counterterms since the gravity action typically diverges [17, 18, 19, 20]. In [18] for example, it was shown that the full (Euclidean) gravitational action in $D=d+1$ spacetime dimensions has three contributions

$$
I_{A d S}=I_{b u l k}\left(g_{\alpha \beta}\right)+I_{\text {surf }}\left(g_{\alpha \beta}\right)+I_{c t}\left(h_{m n}\right),
$$

where $I_{b u l k}$ is the familiar classical action given by

$$
I_{\text {bulk }}=-\frac{1}{2 \ell_{P}^{d-1}} \int_{\mathcal{M}} d^{d+1} x \sqrt{g}\left(R+\frac{d(d-1)}{L^{2}}\right),
$$

$I_{\text {surf }}$ is the Gibbons-Hawking term given by

$$
I_{\text {surf }}=-\frac{1}{\ell_{P}^{d-1}} \int_{\partial \mathcal{M}} d^{d} x \sqrt{h} K
$$

\footnotetext{
${ }^{1}$ In fact there were two different actions proposed in [6] only one of which agrees with the AdS/CFT analysis.
} 
with $K=h^{m n} \nabla_{m} \hat{n}_{n}$ being the trace of the extrinsic curvature of the boundary. Here $h_{m n}$ is the induced metric on the boundary defined through $h_{m n}=g_{m n}-\hat{n}_{m} \hat{n}_{n}$ with $\hat{n}$ being an outward pointing unit normal vector to the boundary. The counterterm action $I_{c t}$ can be arranged as an expansion in powers of the boundary curvature [18]:

$$
\begin{aligned}
I_{c t}=\frac{1}{\ell_{P}^{d-1}} \int_{\partial \mathcal{M}} d^{d} x \sqrt{h}\left[\frac{d-1}{L}+\frac{L}{2(d-2)} \mathcal{R}\right. \\
\left.\quad+\frac{L^{3}}{2(d-4)(d-2)^{2}}\left(\mathcal{R}_{\mu \nu} \mathcal{R}^{\mu \nu}-\frac{d}{4(d-1)} \mathcal{R}^{2}\right)+\cdots\right],
\end{aligned}
$$

where $\mathcal{R}$ and $\mathcal{R}_{\mu \nu}$ are the Ricci scalar and Ricci tensor made out of $h_{\mu \nu}$. Our interest in this paper is $d=3$ in other words the counterterms in $\mathrm{AdS}_{4}$. Notice that the four derivative terms in eq.(44) take the form

$$
\mathcal{R}_{\mu \nu} \mathcal{R}^{\mu \nu}-\frac{3}{8} \mathcal{R}^{2}
$$

which is precisely what arises in new massive gravity! Let us review how this comes about in the analysis of [18]. AdS metrics can be written as

$$
d s^{2}=\frac{d r^{2}}{k+\frac{r^{2}}{L^{2}}}+\left(k+\frac{r^{2}}{L^{2}}\right) d \hat{\Sigma}_{-k, \hat{m}}^{2}+\frac{r^{2}}{L^{2}} d \tilde{\Sigma}_{k, \tilde{m}}^{2}
$$

where $k=0, \pm 1$. The metrics $d \hat{\Sigma}_{-k, \hat{m}}^{2}$ and $d \tilde{\Sigma}_{k, \tilde{m}}^{2}$ are defined through

$$
d \Sigma_{k, m}^{2}= \begin{cases}L^{2} d \Omega_{m}^{2} & \text { for } k=+1 \\ \sum_{i=1}^{m} d x_{i}^{2} & \text { for } k=0 \\ L^{2} d \Psi_{m}^{2} & \text { for } k=-1\end{cases}
$$

where $d \Omega_{m}^{2}$ is the metric on a unit $m$-sphere and $d \Psi_{m}^{2}$ is the metric on a $m$-hyperboloid whose metric is obtained by the analytic continuation of the metric on the unit $m$-sphere. In this way $\mathrm{AdS}_{4}$ can be written such that the boundary is on $R^{3}, S^{3}, H^{3}, S^{2} \times S^{1}, H^{2} \times S^{1}$. Consider the case when the boundary is $S^{3}$. To derive the above form for $I_{c t}$ let us begin with

$$
I_{c t}=\frac{1}{\ell_{P}^{2}} \int_{\partial \mathcal{M}} d^{3} x \sqrt{h}\left(\frac{\kappa}{L}+\mu L \mathcal{R}+L^{3}\left(\lambda_{1} \mathcal{R}_{\mu \nu} \mathcal{R}^{\mu \nu}+\lambda_{2} \mathcal{R}^{2}\right)\right) .
$$

Then integrating $r$ between 0 and $\Lambda$, with $\Lambda$ being a cut-off, we find

$$
I_{\text {bulk }}+I_{\text {surf }}=2 \frac{\Lambda^{2} V_{S^{3}}}{\ell_{P}^{2}}\left(1+\frac{\Lambda^{2}}{L^{2}}\right)^{3 / 2}
$$

The square-root form of the result will give us motivation to consider a compact all-order expression for the counterterm to be made precise shortly. For the moment, we find that expanding around $\Lambda \rightarrow \infty$ gives us

$$
\frac{I_{A d S, S^{3}}}{V_{S^{3}}}=\frac{\Lambda^{3}}{L \ell_{P}^{2}}(2-\kappa)+\frac{\Lambda L}{\ell_{P}^{2}}(3-6 \mu)+\frac{3 L^{3}}{4 \ell_{P}^{2} \Lambda}\left(1-16 \lambda_{1}-48 \lambda_{2}\right)+\cdots .
$$

We can do a similar analysis for $S^{1} \times S^{2}$ (or $S^{1} \times H^{2}$ ) which gives us

$$
\frac{I_{A d S, S^{1} \times S^{2}}}{V_{S^{1} \times S^{2}}}=\frac{\Lambda^{3}}{L \ell_{P}^{2}}(2-\kappa)+\frac{\Lambda L}{2 \ell_{P}^{2}}(\kappa-4+4 \mu)+\frac{L^{3}}{8 \ell_{P}^{2} \Lambda}\left(\kappa-16 \lambda_{1}-32 \lambda_{2}-8 \mu\right)+\cdots .
$$


Then demanding that the $\Lambda^{3}$ and $\Lambda$ divergences cancel gives us $\kappa=2, \mu=1 / 2$. Now let us demand that the final answer is independent of the cut-off $\Lambda$. This means that the $1 / \Lambda$ terms should also cancel. This leads to $\lambda_{1}=-1 / 2, \lambda_{2}=3 / 16$ which is what eq.(4) has. This form for $I_{c t}$ makes the result cut-off independent upto $O(1 / \Lambda)$ for any form for AdS allowed by eq.(6).

The square-root form for $I_{\text {bulk }}+I_{\text {surf }}$ for the $S^{3}$ case makes it very tempting to conjecture a square-root form for the counterterm action. In particular, let us consider

$$
I_{c t}=\beta_{1} \sqrt{-\operatorname{det}\left(\mathcal{R}_{\mu \nu}+\alpha_{1} \mathcal{R} h_{\mu \nu}+\frac{\alpha_{2}}{L^{2}} h_{\mu \nu}\right)} .
$$

Mann in [21] considered a similar counterterm without the $\mathcal{R}_{\mu \nu}$ to remove divergences in $\mathrm{AdS}_{4}$. We find that if the total action $I_{A d S}$ is cut-off independent to all orders in $\Lambda$ for $S^{3}$ then there are two choices:

Either

$$
\alpha_{1}=-\frac{1}{2}, \quad \beta_{1}=-\frac{2 L^{2}}{\ell_{P}^{2}}, \quad \alpha_{2}=-1,
$$

or

$$
\alpha_{1}=-\frac{1}{6}, \quad \beta_{1}=\frac{2 i L^{2}}{\ell_{P}^{2}}, \quad \alpha_{2}=1
$$

While the first choice gives us precisely the DBI gravity action proposed in [6], the second on 2 is also an action proposed in [6] to give NMG upto quadratic order. The actions in [6] have an extra free coupling which we will address in a moment. This seems like a remarkable coincidence! A comment we would like to make here is that we do not have the freedom to add terms like $\mathcal{R}_{\rho \sigma} \mathcal{R}^{\rho \sigma} h_{\mu \nu}, \mathcal{R}^{2} h_{\mu \nu}$ or $\mathcal{R}_{\mu \rho} \mathcal{R}^{\rho \sigma} h_{\sigma \nu}$ to eq.(12) since these terms behave like $1 / r^{2}$ and would spoil the exact cancellation of the cut-off dependence.

Performing the same analysis for $S^{1} \times S^{2}$ selects eq.(13) as the only set of parameters. As shown in [6, 7] this choice is also consistent with the $c$-theorem method of extending the NMG actions to higher orders in [2]. The counterterm eq.(12) with eq.(13) give us a cut-off independent result for any metric of the form in eq.(6).

If we look at the counterterm action in eq.(12), it is tempting to attempt a field redefinition by which we can set

$$
\tilde{h}_{\mu \nu}=\mathcal{R}_{\mu \nu}-\frac{1}{2} \mathcal{R} h_{\mu \nu}-\frac{1}{L^{2}} h_{\mu \nu} .
$$

This would reset the entire counterterm action to the non-dynamical boundary cosmological constant term. Since $h_{\mu \nu}$ is the boundary value of the bulk metric, this boundary field redefinition would need to arise from a more complicated bulk field redefinition 3 which would make the bulk action higher derivative. Hence we will refrain from making this redefinition.

At this point, one can ask if these counterterms give rise to sensible thermodynamics for AdS black holes even in the presence of a cut-off. It would be a strong check if we can show that the Bekenstein-Hawking area law is still satisfied in a cut-off independent manner with this choice of counterterm. This must be true as the bulk action is just Einstein-Hilbert with a cosmological constant. A straightforward, if somewhat tedious, calculation shows that the on-shell action is cut-off dependent. This is actually expected since in the free energy expression, entropy times temperature appears. If the temperature is cut-off dependent, as turns out to be the case, it must be that to have a cut-off independent entropy, the free energy and hence the action must be cut-off dependent. At this stage, it is essential to realize that the definition of temperature needs some care. The point is that we need to use a coordinate system that ensures that the

${ }^{2}$ The second choice is presumably relevant for de Sitter spaces.

${ }^{3}$ It is not clear if a local bulk field redefinition leading to eq.(15) exists. 
speed of light of the boundary theory (with cut-off) is unity. This entails redefining the time coordinate by a cut-off dependent factor which in turn renormalizes the temperature. Once this is taken into account, we indeed find that the entropy is given by the Bekenstein-Hawking area law as it should! Let us illustrate this in more detail for the spherical case. We write the metric as

$$
d s^{2}=\frac{d r^{2}}{1+f(r)}+g(r)\left(d \theta^{2}+\sin ^{2} \theta d \phi^{2}\right)+(1+f(r)) N d t^{2},
$$

where we have put in a factor of $N$ in front of $d t^{2}$. This factor is chosen to be $g(\Lambda) /[1+f(\Lambda)]$ to ensure that the speed of light is unity in the cut-off field theory. Here $f(r)=r^{2} / L^{2}-\mu / r$ and $g(r)=r^{2}$. We can write $\mu=r_{0}+r_{0}^{3} / L^{2}$ with $r_{0}$ being the location of the horizon. Then the temperature works out to be

$$
T=\frac{3 r_{0}^{2}+L^{2}}{4 \pi r_{0} L^{2}} \sqrt{N}
$$

Then we proceed as usual by defining the free energy through $W=T I_{E}$ and entropy through $S=-\partial W / \partial T=-\partial_{r_{0}} W / \partial_{r_{0}} T$ where we are holding the cut-off fixed. This readily gives

$$
S=\frac{8 \pi^{2} r_{0}^{2}}{\ell_{P}^{2}}=\frac{A}{4 G}
$$

Without the crucial $N$ dependent factor in the definition of temperature, one would not get this expected result unless we are in the $\Lambda=\infty$ limit. This computation therefore demonstrates that our counterterm is indeed doing its role as expected in the computation of the BekensteinHawking entropy. Writing the entropy density as $s=c_{S} T^{2}$ then would imply that the effective number of degrees of freedom, $c_{S}$, depends on the cut-off.

Let us now ask the question as to what happens if $I_{B I}$ was treated as a stand-alone action rather than a counterterm. In the context of AdS/CFT, $I_{B I}$ will be dual to a $1+1 \mathrm{CFT}$. The action proposed in [6] in Euclidean signature consists of two pieces

$$
I_{B I}=-\frac{4 m^{2}}{\kappa^{2}} \int d^{3} x\left[\sqrt{-\operatorname{det}\left(\frac{\mathcal{G}}{m^{2}}\right)}-\left(\frac{\lambda}{2 m^{2}}+1\right) \sqrt{\operatorname{det} h}\right],
$$

where $\mathcal{G}_{\mu \nu}=\mathcal{R}_{\mu \nu}-\frac{1}{2} h_{\mu \nu} \mathcal{R}-m^{2} h_{\mu \nu}$. In order for the weak field expansion to be consistent with eq.(41), we need to set $\lambda=2 / L^{2}$. But then comparing with eq.(12) and eq.(13) we have $\left(\frac{\lambda}{2 m^{2}}+1\right)=0$. As shown in [7], the central charge of the $1+1$ CFT is proportional to $\left(\frac{\lambda}{2 m^{2}}+1\right)$. Thus the choice of parameters in eq.(13) in the AdS/CFT context corresponds to having a dual CFT with zero central charge! Of course this only makes sense if we treated this action as a stand-alone action in the context of $\mathrm{AdS}_{3} / \mathrm{CFT}_{2}$ and not as a counterterm to $\mathrm{AdS}_{4}$. Further, the mass of the massive mode has been pushed to zero 4 . It will be interesting to consider fluctuations of the boundary metric along the lines of [22] with Neumann boundary conditions.

One important comment about the stand-alone $c=0$ DBI-NMG action is the following. Consider doing the field redefinition in eq.(15). Naively this would appear to make the theory trivial. However, the field redefinition cannot trivialise this action. In order to see this, consider the path integral representation for this theory. While the field redefinition in eq.(15) would reduce the action to a trivial volume element, the change in the integration measure will give rise to a Jacobian factor. Since the field redefinition involves derivatives of the metric tensor, the Jacobian when put back in the exponent will reinstate the dynamics into the new action.

\footnotetext{
${ }^{4}$ In a previous version, it was erroneously stated that the mass is pushed to infinity. We thank B. Tekin for pointing this out.

${ }^{5}$ We thank Rob Myers for pointing this out to us.
} 
It is well known that three dimensional gravity can be written in terms of a Chern-Simons gauge theory [15, 16]. The one form gauge field is a linear combination of spin connection $\omega_{a b}$ and dreibein,

$$
A^{a \pm}=\omega^{a} \pm \frac{1}{\ell} e^{a}
$$

where $\omega^{a}=\epsilon^{a b c} \omega_{b c} / 2$ is dualised spin connection and $e$ is dreibein and $a$ is a gauge index corresponding to $\mathrm{SL}(2, \mathrm{R}) 6$. Note that $a$ runs from 1 to 3 which coincides with the number of spacetime dimensions. The field strength corresponding to this gauge field can then be related to the Riemann curvature subject to metric compatibility of the connection,

$$
F^{a \pm}=\frac{1}{2} \epsilon^{a b c} R^{b c}+\frac{1}{2 \ell^{2}} \epsilon^{a b c} e^{b} \wedge e^{c} \pm \frac{1}{\ell}\left(d e^{a}+\epsilon^{a b c} \omega^{b} \wedge e^{c}\right)
$$

The metric compatibility condition in terms of dreibein and spin connection is the torsion free constraint

$$
d e^{a}+\epsilon^{a b c} \omega^{b} \wedge e^{c}=0 .
$$

Once we impose the torsion free constraint, the field strengths for both gauge fields is identical because before imposing the constraint they differ by the torsion constraint. For later purposes it is appropriate to define the field strength

$$
\mathcal{F}^{a}=\frac{1}{2}\left(F^{a+}+F^{a-}\right)
$$

$\mathcal{F}_{\mu \nu}^{a}$ does not contain the torsion constraint and therefore is suitable for expressing the curvature tensor in terms of gauge field strength. We will be using eq.(23) to rewrite the DBI-NMG action in terms of gauge fields. To do that we note the following identity in 3 dimensions [23]

$$
G_{\nu}^{\mu}=-\frac{1}{4} \varepsilon^{\mu \rho \sigma} \varepsilon_{\nu \lambda \kappa} R_{\rho \sigma}^{\lambda \kappa} .
$$

Since we are interested in writing all geometric quantities in terms of gauge fields, we will use eq.(21) and eq.(23) to express the Einstein tensor in terms of the gauge field strength $\mathcal{F}_{\mu \nu}^{a}$

$$
G_{\nu}^{\mu}=-\frac{1}{4} \varepsilon^{\mu \rho \sigma} \varepsilon_{\nu \lambda \kappa}\left(\epsilon^{a b c} \mathcal{F}_{\rho \sigma}^{a} e^{b \lambda} e^{c \kappa}-\frac{1}{\ell^{2}} \delta_{\rho}^{\lambda} \delta_{\sigma}^{\kappa}\right) .
$$

It is more instructive to write the Einstein tensor with mixed indices

$$
G^{a \mu}=-\frac{1}{2 \sqrt{\operatorname{det} h}} \varepsilon^{\mu \nu \rho}\left(\mathcal{F}_{\nu \rho}^{a}-\frac{1}{\ell^{2}} \epsilon^{a b c} e_{b \nu} e_{c \rho}\right) .
$$

This equation can be further rearranged to write the gauge field strength in terms of geometric quantities, namely the dreibeins and the Einstein tensor,

$$
G^{a \mu}-\frac{1}{2 \ell^{2} \sqrt{\operatorname{det} h}} \varepsilon^{\mu \nu \rho} \epsilon^{a b c} e_{b \nu} e_{c \rho}=-\frac{1}{2 \sqrt{\operatorname{det} h}} \varepsilon^{\mu \nu \rho} \mathcal{F}_{\nu \rho}^{a} \equiv \frac{1}{\sqrt{\operatorname{det} h}} \star \mathcal{F}^{a \mu},
$$

using which we find

$$
\sqrt{-\operatorname{det}(\mathcal{G})}=\sqrt{\operatorname{det} \star \mathcal{F}^{a \mu}} .
$$

The dual gauge field strength carries mixed indices, however as mentioned before, in three dimensions, gauge and spacetime indices have same range and therefore the determinant of

\footnotetext{
${ }^{6}$ We follow the conventions in [23] and these are summarized in the appendix.
} 
dual field strength is a sensible quantity to consider. In fact, it can be written in a more suggestive form as

$$
\sqrt{-\operatorname{det}(\mathcal{G})}=\sqrt{\operatorname{det} \star \mathcal{F}}=\sqrt{\frac{1}{6} \varepsilon^{\mu \nu \rho} \epsilon^{a b c} \star \mathcal{F}_{a \mu} \star \mathcal{F}_{b \nu} \star \mathcal{F}_{c \rho}}
$$

While the lagrangian density, in terms of the metric, reproduces the lagrangian density of the new massive gravity in the weak field expansion, there is no such expansion available in the gauge field formulation.

At this point, it is interesting to note that we can write a more general action by adding a Chern-Simons action to this gauge theory action:

$$
\begin{aligned}
I_{\text {gen }} & =\xi \int d^{3} x \operatorname{Tr}\left[A^{+} \wedge d A^{+}+A^{+} \wedge A^{+} \wedge A^{+}\right. \\
& \left.-A^{-} \wedge d A^{-}-A^{-} \wedge A^{-} \wedge A^{-}\right]+\eta \int d^{3} x \sqrt{\operatorname{det} \star \mathcal{F}}
\end{aligned}
$$

We can express (30) in terms of the gravity variables,

$$
I_{\text {gen }}=\frac{\xi}{\ell} \int d^{3} x \sqrt{\operatorname{det} h}\left[R+\frac{1}{\ell^{2}}\right]+\eta \int d^{3} x \sqrt{-\operatorname{det} \mathcal{G}} .
$$

This would correspond to a $1+1$ CFT with $c \neq 0$ for non-vanishing $\xi$.

In this letter, we have found that the DBI extension of new massive gravity can arise as a counterterm in $\mathrm{AdS}_{4}$. This choice makes the zero temperature action cut-off independent and gives a sensible area law for the Schwarzschild black hole. Let us now comment on possible generalizations to higher dimensions. If one considers odd bulk dimensions, then the dual CFT is even dimensional and has conformal anomalies. These manifest themselves as logarithmic terms in the on-shell bulk action evaluated for non-trivial boundary topologies. There are no local counterterms which can remove these logarithmic divergences and it is not apriori clear that the magic in $3+1$ dimensions can be extended to odd dimensional bulk theories. We leave the examination of higher even bulk dimensions as an interesting open problem. It will also be interesting to work out the counterterms in the presence of higher derivative terms in the bulk action (see for example [24] for interesting actions with higher derivative terms) to see if there is a general lesson to be learnt.

Finally let us comment on the relation between recent work on holographic renormalization group flows [25, 26, 27, 28]. These attempt at putting the boundary at a finite radius and integrating out all the degrees of freedom associated with larger values of radius. Schematically the total action is written as [28]

$$
S=\int_{r<\Lambda} d^{d+1} x \sqrt{g} \mathcal{L}+S_{B}
$$

where $S_{B}$ is a boundary action defined on $r=\Lambda$ and can be viewed as a boundary state for the bulk theory in the region $r<\Lambda$. The choice of the cut-off is arbitrary and the on-shell action is independent of the cut-off in case of pure AdS bulk. However, in case of geometries which asymptote to AdS, the on-shell action is cut-off dependent but it is just as well, because the cut-off dependence of the on-shell action as well as the local temperature is such that it correctly reproduces the Bekensetin-Hawking entropy in case of AdS-black hole backgrounds. In what we are doing $S_{B}$ is the sum of the Gibbons-Hawking term and the counterterm. Since we have found the total action to be cut-off independent for $T=0$, we have effectively solved the flow equations in [28, for gravity to leading order in the context of $\mathrm{AdS}_{4} / \mathrm{CFT}_{3}$. 
To understand utility of these counterterms, it is instructive to look at them from the boundary field theory point of view7. In the field theory, one defines a set of local counterterms once and for all, irrespective of what vacuum one is working with. One could then be working with a cut-off field theory or with a finite temperature field theory-this does not affect the local counterterms. For example, the Euclidean description of a finite temperature field theory corresponds to making the Euclideanised time coordinate periodic. This global periodicity condition does not affect the form of the local counterterms. As a result even thermal field theories will have the same set of local counterterms. Of course, one has to ask appropriate questions about physical observables in such theories. We have looked at this problem from the bulk side and have demonstrated that the Bekenstein-Hawking entropy of the black hole in AdS space can be recovered if we define the local temperature at the cut-off surface appropriately. In the same vein, it would be interesting to find a prescription for correlation functions of physical observables. We will leave this analysis for future work.

Acknowledgments : We would like to thank P. Argyres, G. Arutyunov, N. Banerjee, S. R. Das, B. de Wit, S. Dutta, R. K. Gupta, J. Hung, M. Paulos, M. Rangamani, A. Shapere, B. Tekin, S. Theisen and S. Vandoren for discussions. In particular, we would like to thank R. Myers and A. Sen for useful suggestions and discussions and R. Myers, M. Rangamani and B. Tekin for comments on the draft. We would like to thank Perimeter Institute for hospitality during this work. D.P.J would like to thank University of Kentucky, Utrecht University, Albert Einstein Institute and Indian Institute of Science for hospitality during the course of this work. A.S. thanks Princeton University for hospitality where part of this work was presented. We thank the organizers for ISM2011 for hospitality and an opportunity to present this work.

\section{Appendix}

Here we will summarise our conventions. We will be working in the Euclidean signature. We use indices $\mu, \nu, \rho, \cdots$ to denote three dimensional and $a, b, c, \cdots$ to denote gauge/local Lorentz indices in three dimensions. Indices $\alpha, \beta, \cdots$ are general $d$-dimensional indices.

The $S L(2, R)$ generators are given by

$$
T^{0}=\frac{1}{2}\left(\begin{array}{cc}
0 & -1 \\
1 & 0
\end{array}\right), T^{1}=\frac{1}{2}\left(\begin{array}{cc}
1 & 0 \\
0 & -1
\end{array}\right), T^{2}=\frac{1}{2}\left(\begin{array}{cc}
0 & 1 \\
1 & 0
\end{array}\right),
$$

such that

$$
\operatorname{Tr}\left(T^{a} T^{b}\right)=\frac{1}{2} \eta^{a b}, \quad\left[T^{a}, T^{b}\right]=\epsilon^{a b c} T^{c},
$$

where, $\epsilon^{012}=1$. The group indices $a, b, c, \cdots$ are raised and lowered by the flat Lorentzian metric $\eta_{a b}$. The Levi-Civita tensor $\varepsilon_{\mu \nu \rho}$ is totally antisymmetric tensor with \pm 1 and 0 entries. The Levi-Civita tensor density will be written in terms of $\varepsilon_{\mu \nu \rho}$ with explicit insertions of $\sqrt{\operatorname{det} h}$.

\section{References}

[1] E. A. Bergshoeff, O. Hohm and P. K. Townsend, "Massive Gravity in Three Dimensions," Phys. Rev. Lett. 102, 201301 (2009) [arXiv:0901.1766 [hep-th]].

[2] A. Sinha, "On the new massive gravity and AdS/CFT," JHEP 1006, 061 (2010) arXiv:1003.0683 [hep-th]].

\footnotetext{
${ }^{7}$ We thank Ashoke Sen for discussions on this issue.
} 
[3] R. C. Myers and A. Sinha, "Seeing a c-theorem with holography," Phys. Rev. D 82, 046006 (2010) arXiv:1006.1263 [hep-th]].

A. Sinha, "On higher derivative gravity, c-theorems and cosmology," arXiv:1008.4315 [hepth].

R. C. Myers and A. Sinha, "Holographic c-theorems in arbitrary dimensions," arXiv:1011.5819 [hep-th].

J. T. Liu, W. Sabra and Z. Zhao, "Holographic c-theorems and higher derivative gravity," arXiv:1012.3382 [hep-th].

[4] M. F. Paulos, "New massive gravity, extended," arXiv:1005.1646 [hep-th].

[5] S. Deser and G. W. Gibbons, "Born-Infeld-Einstein actions?," Class. Quant. Grav. 15, L35 (1998) arXiv:hep-th/9803049.

[6] I. Gullu, T. Cagri Sisman and B. Tekin, "Born-Infeld extension of new massive gravity," Class. Quant. Grav. 27, 162001 (2010) arXiv:1003.3935 [hep-th]].

[7] I. Gullu, T. C. Sisman and B. Tekin, "c-functions in the Born-Infeld extended New Massive Gravity," Phys. Rev. D 82, 024032 (2010) [arXiv:1005.3214 [hep-th]].

[8] A. Ghodsi and D. M. Yekta, "Black Holes in Born-Infeld Extended New Massive Gravity," arXiv:1010.2434 [hep-th].

M. Alishahiha, A. Naseh and H. Soltanpanahi, "On Born-Infeld Gravity in Three Dimensions," Phys. Rev. D 82, 024042 (2010) arXiv:1006.1757 [hep-th]].

[9] Y. Liu and Y. W. Sun, "Note on New Massive Gravity in $A d S_{3}$," JHEP 0904, 106 (2009) arXiv:0903.0536 [hep-th]].

Y. Liu and Y. W. Sun, "Consistent Boundary Conditions for New Massive Gravity in $A d S_{3}, "$ JHEP 0905, 039 (2009) arXiv:0903.2933 [hep-th]].

[10] E. A. Bergshoeff, O. Hohm and P. K. Townsend, "More on Massive 3D Gravity," Phys. Rev. D 79, 124042 (2009) arXiv:0905.1259 [hep-th]].

[11] I. Gullu, T. C. Sisman and B. Tekin, "All Bulk and Boundary Unitary Cubic Curvature Theories in Three Dimensions," arXiv:1011.2419 [hep-th].

[12] D. Grumiller and O. Hohm, "AdS3/LCFT2 - Correlators in New Massive Gravity," Phys. Lett. B 686, 264 (2010) [arXiv:0911.4274 [hep-th]].

[13] D. Grumiller, N. Johansson and T. Zojer, "Short-cut to new anomalies in gravity duals to logarithmic conformal field theories," arXiv:1010.4449 [hep-th].

[14] M. R. Gaberdiel, D. Grumiller and D. Vassilevich, "Graviton 1-loop partition function for 3-dimensional massive gravity," arXiv:1007.5189 [hep-th].

[15] A. Achucarro and P. K. Townsend, "A Chern-Simons Action for Three-Dimensional anti-De Sitter Supergravity Theories," Phys. Lett. B 180, 89 (1986).

[16] E. Witten, "(2+1)-Dimensional Gravity as an Exactly Soluble System," Nucl. Phys. B 311, 46 (1988).

[17] V. Balasubramanian and P. Kraus, "A stress tensor for anti-de Sitter gravity," Commun. Math. Phys. 208, 413 (1999) arXiv:hep-th/9902121.

[18] R. Emparan, C. V. Johnson and R. C. Myers, "Surface terms as counterterms in the AdS/CFT correspondence," Phys. Rev. D 60, 104001 (1999) arXiv:hep-th/9903238.

[19] P. Kraus, F. Larsen and R. Siebelink, "The gravitational action in asymptotically AdS and flat spacetimes," Nucl. Phys. B 563, 259 (1999) arXiv:hep-th/9906127. 
[20] S. de Haro, S. N. Solodukhin and K. Skenderis, "Holographic reconstruction of spacetime and renormalization in the AdS/CFT correspondence," Commun. Math. Phys. 217, 595 (2001) arXiv:hep-th/0002230.

[21] R. B. Mann, "Entropy of rotating Misner string spacetimes," Phys. Rev. D 61, 084013 (2000) arXiv:hep-th/9904148.

[22] G. Compere, D. Marolf, "Setting the boundary free in AdS/CFT," Class. Quant. Grav. 25, 195014 (2008). arXiv:0805.1902 [hep-th]].

[23] S. Carlip, "Conformal field theory, (2+1)-dimensional gravity, and the BTZ black hole," Class. Quant. Grav. 22, R85 (2005) arXiv:gr-qc/0503022.

[24] A. Buchel, R. C. Myers, M. F. Paulos and A. Sinha, "Universal holographic hydrodynamics at finite coupling," Phys. Lett. B 669, 364 (2008) [arXiv:0808.1837 [hep-th]].

A. Adams, A. Maloney, A. Sinha and S. E. Vazquez, "1/N Effects in Non-Relativistic Gauge-Gravity Duality," JHEP 0903, 097 (2009) [arXiv:0812.0166 [hep-th]].

A. Buchel, R. C. Myers and A. Sinha, "Beyond eta/s = 1/4pi," JHEP 0903, 084 (2009) arXiv:0812.2521 [hep-th]].

R. C. Myers, M. F. Paulos and A. Sinha, "Holographic Hydrodynamics with a Chemical Potential," JHEP 0906, 006 (2009) arXiv:0903.2834 [hep-th]].

A. Buchel, J. Escobedo, R. C. Myers, M. F. Paulos, A. Sinha and M. Smolkin, "Holographic GB gravity in arbitrary dimensions," JHEP 1003, 111 (2010) [arXiv:0911.4257 [hep-th]].

[25] I. Bredberg, C. Keeler, V. Lysov and A. Strominger, "Wilsonian Approach to Fluid/Gravity Duality," arXiv:1006.1902 [hep-th].

[26] D. Nickel and D. T. Son, "Deconstructing holographic liquids," arXiv:1009.3094 [hep-th].

[27] I. Heemskerk and J. Polchinski, "Holographic and Wilsonian Renormalization Groups," arXiv:1010.1264 [hep-th].

[28] T. Faulkner, H. Liu and M. Rangamani, "Integrating out geometry: Holographic Wilsonian RG and the membrane paradigm," arXiv:1010.4036 [hep-th]. 